\title{
Single photon nonlinear optics on photonic crystal chips
}

Dirk Englund, Andrei Faraon, Ilya Fushman, and Jelena Vučković

A single semiconductor quantum dot that is strongly coupled to a photonic crystal nanocavity yields optical nonlinearities at the single photon level.

The promise of quantum information processing is driving developments in several different physical implementations. Photons are attractive as carriers of quantum information because they can travel long distances without losing their information through interactions with their environment. However, their passive nature can be a problem in applications such as the construction of logic gates, where it is necessary for two or more photons to interact. This interaction requires a nonlinear optical medium. Particularly strong nonlinearities are achieved when photons drive an emitter near its resonance frequency, and this nonlinearity is even greater if the photon is confined near the emitter in an optical cavity.

We work with single semiconductor quantum dots (QDs) coupled to photonic crystal nanocavities. Recently, we showed that such a system enables an optical nonlinearity ${ }^{1}$ at the single-photon level: the cavity transmission spectrum changes dramatically when the power nears one intracavity photon number $\left\langle n_{\text {cav }}\right\rangle$ per cavity lifetime (see Figure 1 ). The transmission spectrum obtained in the cross-polarized configuration shown in Figure 1(a) changes as plotted in Figure 1(b) when $\left\langle n_{\text {cav }}\right\rangle$ increases from nearly zero to $\left\langle n_{\text {cav }}\right\rangle \sim 5$. This nonlinearity relies on saturation of the QD, which occurs when the photon flux reaches roughly one photon per emitter lifetime.

This single-photon-level nonlinearity has many promising applications. One application of particular interest in quantum information processing concerns the realization of large phase shifts on a signal beam conditioned on the intensity of a control beam. Using a homodyne setup, we measured the phase of the transmission through the cavity near the QD resonance frequency. ${ }^{2}$ As the probe power increases to $\left\langle n_{\text {cav }}\right\rangle \sim 3$ and the QD saturates, we found that the phase of the transmitted beam changes by up to $0.24 \pi$. In applications such as quantum (a)
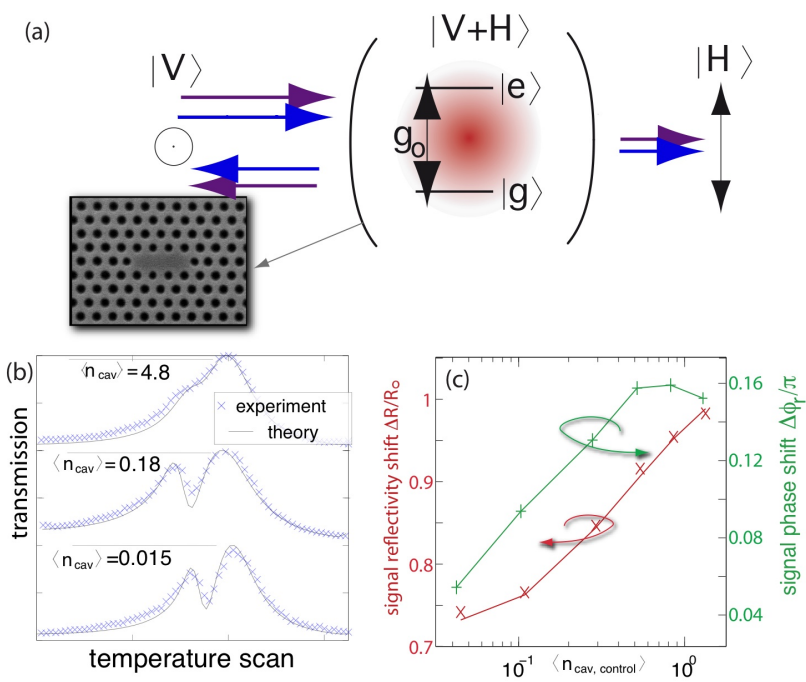

Figure 1. Resonant probing of the quantum dot (QD)-cavity system. (a) The cavity is probed by a vertically $(V)$ polarized laser beam. The transmission of the cavity is detected in the orthogonal polarization $(H)$ in order to reduce direct scattering from the incident beam. We model the quantum dot as an excited (e) and ground state ( $g$ ) which is coupled to the cavity mode by a rate $g$. (b) The transmission spectrum shows the vacuum Rabi splitting due to the strongly coupled $Q D$. The splitting disappears as the QD is saturated at an intracavity photon number $\left\langle n_{\text {cav }}\right\rangle$ near unity. (c) The phase of the signal beam changes by up to $0.16 \pi$ when the control beam intensity is raised to an incident power of $1 \mu W$, corresponding to $\left\langle n_{\text {cav }}\right\rangle \approx 1$.

nondemolition measurements, it is useful to spectrally separate the control and signal beams. Thus, for a detuning of $0.03 \mathrm{~nm}$, we measured a $0.16 \pi$ phase change in the signal beam when the control beam intensity was raised to $\left\langle n_{\text {cav }}\right\rangle \approx 1$, as plotted in Figure 1(c).

A measurement of the photon statistics reveals the quantum nature of the interaction in the strongly coupled QD/cavity system. ${ }^{3}$ One interesting phenomenon is the photon blockade effect, in which light couples to the cavity

Continued on next page 


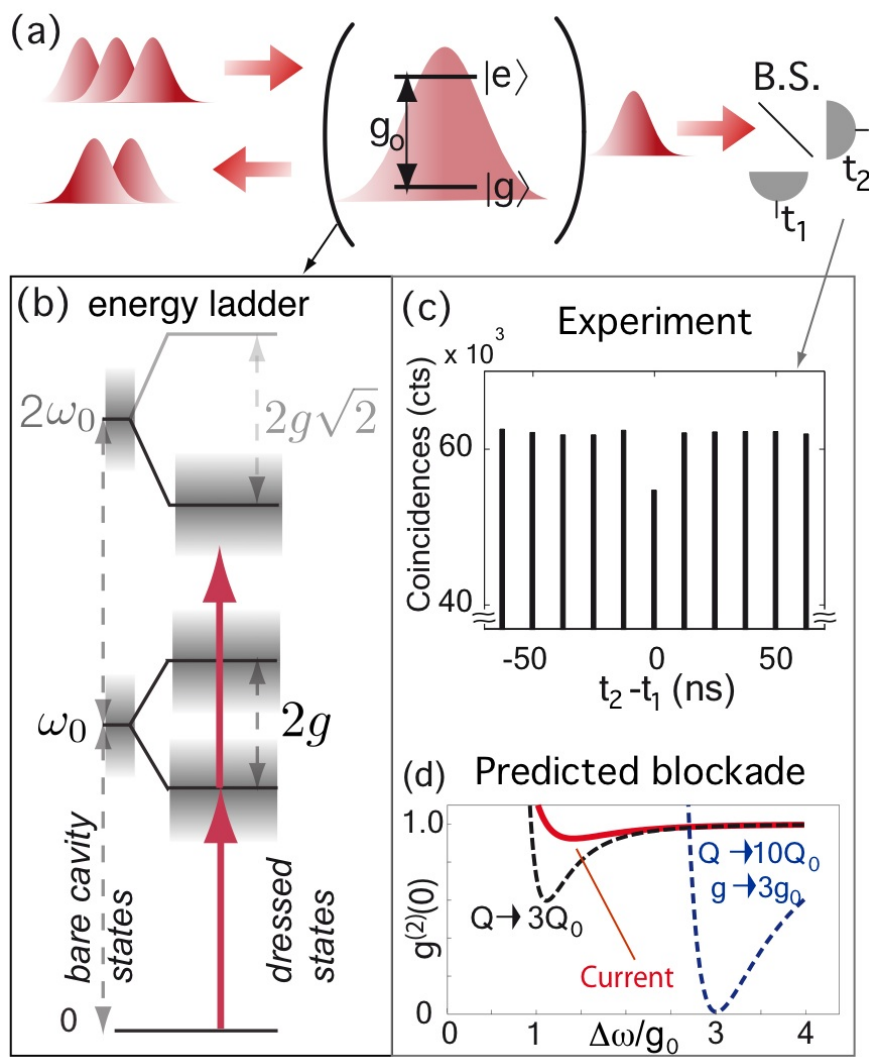

Figure 2. (a) The statistics of the outgoing pulse were measured by a Hanbury-Brown/Twiss setup, consisting of a beam splitter followed by two photodetectors. Clicks on the photodetectors are recorded as a function of the time delay $t 2-t 1$. (b) The Jaynes-Cummings ladder of energy states (right) is anharmonic, in contrast to the bare cavity (left), which has a frequency spacing of $\omega_{0}$. (c) Experimental observation of antibunching in the transmitted light field to $g^{(2)}(0) \approx 0.92$, which is estimated by the drop in coincidence counts between detectors 1 and 2 at time delay zero. (d) Theoretical prediction of the blockade as a function of the probe detuning from the cavity resonance when the quality factor, $Q$, and the $Q D$-cavity coupling rate, $g$, are increased over their current values $Q_{0}$ and $g_{0}$.

photon-by-photon, ${ }^{4,5}$ which is analogous to the Coulomb blockade of electrons. ${ }^{6}$ The photon blockade occurs because of the anharmonicity of the Jaynes-Cummings ladder of energy eigenstates. ${ }^{4}$ Figure 2(a) illustrates this phenomenon: one photon (red arrow) reaches the first photon manifold, but a second photon is detuned from the next transition. As a result, we observed photon anti-bunching in the photon statistics of a transmitted beam: see Figure 2(c). In other words, the probability that multiple photons pass through within a cavity decay time is reduced in transmitted light as compared to classical light. Our measurement of the anti-bunching is less pronounced than a similar result using neutral atoms ${ }^{7}$ because the splitting of the levels is on the same order as the cavity linewidth. Figure 2(b) indicates this with the blurring of the energy levels. However, if the quality factor $(Q)$ were increased by a factor of 3 , the anti-bunching at zero time delay, $g^{(2)}(0)$, would drop to 0.6 . This is shown in the theoretical prediction in Figure 2(d). Another interesting phenomenon of the strongly coupled QD/cavity system is photon-induced tunneling. In this case, photons tend to pass through in multiples, resulting in photon bunching. ${ }^{3}$

The large nonlinear interaction we have demonstrated is important for scalable quantum communication and computation relying on photonic qubits. In future work, we plan to improve the coupling efficiency and explore applications of the nonlinearity in on-chip photonic networks. ${ }^{8,9}$

Dirk Englund, Andrei Faraon, and Ilya Fushman contributed equally to this article.

\section{Author Information}

\section{Dirk Englund}

Department of Physics

Harvard University

Cambridge, MA

Ginzton Laboratory

Stanford University

Stanford, CA

Dirk Englund received his MS in electrical engineering and $\mathrm{PhD}$ in applied physics from Stanford University in 2008, working with Jelena Vučković as a graduate and postdoctoral student. He is currently a postdoctoral fellow at Harvard University.

\section{Andrei Faraon, Ilya Fushman , and Jelena Vučković Ginzton Laboratory \\ Stanford University \\ Stanford, CA}

Andrei Faraon received his undergraduate degree in physics from Caltech and is currently a graduate student in applied physics, working with Jelena Vučković.

Ilya Fushman received his MS in electrical engineering and $\mathrm{PhD}$ in applied physics from Stanford University in 2008, working with Jelena Vučković. 
Jelena Vučković received her PhD in electrical engineering from Caltech and has been on the faculty at Stanford University since 2003.

\section{References}

1. D. Englund, A. Faraon, I. Fushman, N. Stoltz, P. Petroff, and J. Vučković, Controlling cavity reflectivity with a single quantum dot, Nature 450 (6), pp. 857-61, 2007.

2. I. Fushman, D. Englund, A. Faraon, N. Stoltz, P. Petroff, and J. Vuckovic, Controlled phase shifts with a single quantum dot, Science 320 (5877), pp. 769-772, 2008. doi:10.1126/science. 1154643

3. A. Faraon, I. Fushman, D. Englund, N. Stoltz, P. Petroff, and J. Vuckovic, Coherent generation of nonclassical light on a chip via photon-induced tunneling and blockade, Nature Phys., 2008.

4. L. Tian and H. J. Carmichael, Quantum trajectory simulations of two-state behavior in an optical cavity containing one atom, Phys. Rev. A 46 (11), pp. R6801+, 1992. doi:http://dx.doi.org/10.1103/PhysRevA.46.R6801

5. A. Imamoḡlu, H. Schmidt, G. Woods, and M. Deutsch, Strongly interacting photons in a nonlinear cavity, Phys. Rev. Lett. 79 (8), pp. 1467-1470, 1997.

6. M. Kastner, The single electron transistor and artificial atoms, Ann. Phys. 9, pp. 885894, 2000.

7. K. M. Birnbaum, A. Boca, R. Miller, A. D. Boozer, T. E. Northup, and H. J. Kimble, Photon blockade in an optical cavity with one trapped atom, Nature 436, pp. 87-90, 2005. 8. D. Englund, A. Faraon, B. Zhang, Y. Yamamoto, and J. Vuckovic, Generation and transfer of single photons on a photonic crystal chip, Opt. Express 15, pp. 5550-8, 2007. 9. A. Faraon, I. Fushman, D. Englund, N. Stoltz, P. Petroff, and J. Vuckovic, Dipole induced transparency in waveguide coupled photonic crystal cavities, Opt. Express 16 (16), pp. 12154-12162, 2008. 